\title{
Von links nach rechts: Wandel der regionalen Unterstützung für die technokratisch-populistische Bewegung ANO 2011 bei den tschechischen Parlamentswahlen 2013 und $2017^{1}$
}

\author{
Pavel Maškarinec, Lukáš Novotný
}

Jan-Evangelista-Purkyně-Universität, Ústí nad Labem, Tschechische Republik

maskarinec@centrum.cz, novotny.l@centrum.cz

\section{Zusammenfassung}

Ziel dieser Studie ist es, die Dominanz der ANO-Bewegung insbesondere am Beispiel der zwei Parlamentswahlen von 2013 und 2017 zu erklären und damit zur Diskussion über den „technokratischen Populismus“ beizutragen. Während in Kapitel I der Begriff des technokratischen Populismus erläutert wird, beschäftigt sich das nächste Kapitel mit der Protestbewegung ANO 20 II und deren Erfolgen als Beispiel des technokratischen Populismus. Die Analyse präsentiert Befunde der Parteienforschung sowie der Wahlgeographie und gibt Antworten auf folgende Frage: Überschreitet die räumliche Unterstützung der ANOBewegung angesichts ihrer programmatischen Entideologisierung und ihres technokratisch-kommunikativen Stils die langfristigen räumlichen Trennlinien des WählerInnenspektrums, oder ist sie vorzugsweise in traditionell linken oder rechten Gebieten der Tschechischen Republik erfolgreicher, übernimmt also nur WählerInnen aus den traditionellen Blöcken des traditionellen Parteienspektrums?

\section{Schlüsselwörter}

Populismus, Tschechien, ANO 20II, Andrej Babiš, Wahlgeographie

\section{From the right to the left: transformation of the regional support of the technocratic populist ANO 2011 movement in Czech parliamentary elections of 2013 and 2017}

\begin{abstract}
The aim of this study is to explain the dominance of the ANO movement in the 2013 and 2017 parliamentary election, and thus contribute to the discussion about "technocratic populism". The article opens with a brief overview of the state of research on populism. In particular, the term technocratic populism. The next chapter presents the Czech protest movement ANO $201 \mathrm{I}$ and its successes in the elections as an example of technocratic populism. The following analysis presents selected findings from party research and electoral geography and tries to answer the following question: Given its programmatic deideologization and technocratic communicative style, does the spatial support for the ANO movement cross the long-term spatial dividing lines of Czech party system, or is it more successful in traditionally left or right-wing areas of the Czech Republic, thus only gaining voters from the traditional blocs of the Czech party system?
\end{abstract}

\section{Keywords}

Populism, Czech Republic, ANO 20II, Andrej Babiš

The authors have declared that no competing interests exist.

I Funding: This study was prepared under a grant project supported by the Czech Science Foundation, Grant No. 2O-O455IS, "Patterns of Quality of Democracy at Regional Level in the V4 Countries: Looking Inside the Black Box". 


\section{Einführung}

Die tschechische Regierung mit dem Milliardär Andrej Babiš an der Spitze wettert gern gegen Eliten - schickt ihrerseits jedoch „ExpertInnen“ in die Ministerien. Krisenerscheinungen zwischen „Elite“ und „Basis“ sind (auch) in diesem mitteleuropäischen Land verbreitet, namentlich dienen die alten Parteien nicht mehr hinreichend als Kanäle. Nur etwa jeder fünfte Tschechevertraut laut verschiedenen repräsentativen Meinungsumfragen den Parteien (CVVM 20I9). Die von professionalisierten Parteispitzen ausgehandelte pragmatische und ideologische Politik der Kompromisse wirkt auf viele tschechische WählerInnen als zu distanziert und bürokratisiert. Von dieser Lage profitieren PopulistInnen nicht nur in Tschechien, sondern auch in anderen Ländern des ehemaligen kommunistischen Blocks. Gründe für ihre Erfolge sind die Verengung von Politik auf technokratische Governance, auf deliberative Absprachen zwischen politischen EntscheidungsträgerInnen und demokratisch nicht legitimierten ExpertInnen sowie die vermeintliche Alternativlosigkeit der traditionellen Parteien.

Was treibt die selbsternannten "Retter des Volkes“ um? Diese Frage stellen sich die Politikwissenschaftler und Politikwissenschaftlerinnen immer häufiger (de Vreese et al. 20I8) - auch in diesem Beitrag. Konkret wird sie hier am Beispiel der politischen Bewegung ANO 20 I I und deren Vorsitzenden Andrej Babiš untersucht.

Die ANO-Bewegung wurde 2OII als Protestbewegung gegründet und beteiligt sich bereits seit 2013 an der Regierung. Seit 2017 stellt sie sogar mit Andrej Babiš den Ministerpräsidenten. Nach außen hin zeigt sich ANO äußerst geschickt. Beim tschechischen Innenministerium wurde sie als politische Bewegung, nicht als politische Partei registriert. Hierdurch hat sie die Möglichkeit ihrem Antiparteien- und Anti-Establishment-Appell noch mehr Nachdruck zu verleihen. Andrej Babiš kam als einflussreicher Unternehmer in die Politik, wodurch seine politische Plattform ANO 2OII als Beispiel des politischen Unternehmens, einer business-firm-party (Kopeček 20I6; Brunnerová 20I9) gilt.

Unser Beitrag beginnt mit einem kurzen Überblick über den Forschungsstand zum Populismus. Im Besonderen wird hier der Begriff des technokratischen Populismus (technocratic populism) erläutert. Im nächsten Kapitel wird die Protestbewegung ANO 2OII mit ihren Erfolgen bei den Wahlen seit 2013 als Beispiel des technokratischen Populismus dargestellt. Die nachfolgende Analyse präsentiert ausgewählte Befunde der Parteienforschung sowie der Wahlgeographie und versucht Antworten auf die folgenden Fragen zu geben:

- Überschreitet die räumliche Unterstützung der ANO-Bewegung angesichts ihrer programmatischen Entideologisierung und ihres technokratisch-kommunikativen Stils die langfristigen räumlichen Trennlinien des tschechischen Parteienspektrums oder ist sie vorzugsweise in traditionell linken oder rechten Gebieten der Tschechischen Republik erfolgreicher, übernimmt also nur WählerInnen aus den traditionellen Blöcken des tschechischen Parteienspektrums?

- Was ist die programmatische Besonderheit des technokratischen Populismus der ANO-Bewegung angesichts der Wahlergebnisse von 2013 und 2017 ?

Ziel dieser Studie ist es, die Dominanz der ANO-Bewegung insbesondere am Beispiel der zwei Parlamentswahlen von 2013 und $2017 \mathrm{zu}$ erklären und damit zur Diskussion über den technokratischen Populismus in Ländern des ehemaligen kommunistischen Blocks beizutragen. Hierfür werden neben der räumlichen Dimension, sprich der Wahlerfolge der Partei, auch die Programmatik der ANO-Bewegung sowie der kommunikative Stil von Andrej Babiš untersucht. Mit Hilfe der Methoden der Wahlgeographie soll die Konzentration der Wahlunterstützung in Tschechien dargestellt werden. Auch wird die Annahme überprüft, ob die Unterstützung der zentristischen technokratisch-populistischen Parteien, zu denen beispielsweise auch die ANO-Bewegung gehört (Havlík/Voda 20I8; Havlík 20I9), gleichmäßig im Raum verteilt ist, ohne Rücksicht auf die traditionelle Spaltung des Parteienwettbewerbs, oder ob deren WählerInnenunterstützung ähnliche Muster aufweist, wie sie im tschechischen politischen Wettbewerb auftreten, wonach es Regionen mit traditionell starken rechten oder linken Parteien gibt. Die ANO-Bewegung stellt sich selbst als zentristische Bewegung ohne Ideologie dar. Wir wollen herausfinden, in welchen Regionen sich ihre WählerInnen befinden, worauf die räumliche Unterstützung basiert und welche Trennlinien entscheidend für die Unterstützung dieser Partei sind. Damit füllen unsere Ergebnisse eine Forschungslücke.

\section{Theoretische Überlegungen}

Populismus wird als politische Strategie verstanden, die für das sogenannte „einfache“ Volk und gegen die herrschenden gesellschaftlichen und politischen Eliten Partei ergreift (Stanley 2008). Der Populismusforscher Cas Mudde definiert Populismus als „eine Ideologie, welche die Gesellschaft letztlich in zwei homogene und antagonistische Gruppen unterteilt, ,das reine Volk' gegen die ,korrupte Elite', und argumentiert, dass Politik ein Ausdruck des volonté générale des Volkes sein sollte“ (Mudde 2004, 543). Im Zentrum seiner Definition steht das „Volk“ und die Forderung nach seiner direkten und 
unmittelbaren Herrschaft, der sogenannten „Volkssouveränität" (Müller 20I6), welche die zentrale Idee des Populismus ist.

Wesensmerkmal ist mitunter seine Anti-Establishment-Orientierung, allen voran den etablierten Parteien und PolitikerInnen als den typischen politischen Eliten gegenüber, die der Populismus als korrumpiert ansieht (Rooduijn 20I4). Ein weiterer Aspekt ist die Vorstellung von Homogenität, sowohl der politischen Elite als auch des Volkes, die jeweils als Einheiten ohne Differenzierung nach Gruppen oder Individuen gesehen werden (Canovan 2004; Kaltwasser 20I2). Populistische Parteien artikulieren vernachlässigte Unzufriedenheit. Wir haben es dabei mit einer Widersprüchlichkeit zu tun; „Demokratie“ wird populistisch aufgegriffen. Denn die Populisten nehmen ihrerseits oft ein symbolisches Äquivalent zum Volk in Anspruch, wodurch sie die Demokratie und das Volk repräsentieren. Das Postulat von Homogenität in Kombination mit einem symbolischen Äquivalent schafft also Demokratie im populistischen Sinne (Mouffe 20I6; Canovan 1999; Arditi 2004; 2010). In den jungen Demokratien, wie auch in Tschechien, findet dies Anklang, da die PopulistInnen im Wahlkampf darauf anspielen, dass die alten Eliten korrupt sind und sie die Lösung dafür kennen (technokratische Effizienz) (Buštíková/Guasti 2018).

Die ANO-Bewegung wird hier als technokratischer Populismus eingestuft, welcher die populistische Herangehensweise mit der Expertise verbindet, dass populistische Parteienführer sich in der Exekutive auf Fachleute verlassen und mit ihrer Hilfe versuchen auf das Volk zu wirken und die öffentliche Meinung zu beeinflussen. So zum Beispiel verspricht der politische Leader der ANOBewegung seinen Wählern regelmäßig, den Staat wie eine Firma zu führen, oft mit dem Hinweis darauf, was er in seinem Agrarunternehmen Agrofert seit der politischen Wende von 1989 erreicht hat.

Technokratische PopulistInnen, zu denen wir auch Babiš zählen, suchen sich ExpertInnen aus, die oft keine Mitglieder der entsprechenden Partei oder Bewegung sind und erheben sie als MinisterInnen oder wichtige BeamtInnen in die Exekutive. Somit handeln sie prinzipiell gegen ihre eigene Programmatik, da die politische Macht nicht dem Volk überreicht wird, (de Blasio/Sorice 20I8). Dadurch bekommt das Volk nicht länger die Chance, sich mit öffentlichen Belangen zu beschäftigen.

Die Politik wird im Grunde genommen abgelehnt und die technokratischen PopulistInnen stellen sich damit gegen das Parteiensystem und die Logik der repräsentativen Demokratie (Fischer 1990; Kenneally 2009; Bickerton 2017). Sie stellen sich sogar gegen die Instrumente der direkten Demokratie, wodurch sie von anderen PopulistInnen deutlich zu unterscheiden sind. Dieser Populismus basiert jedoch auch auf einer Anti-ElitenIdeologie, die durch professionelle politische Kommu- nikation Nähe zu den einfachen Menschen inszeniert. Es fällt zudem nicht leicht, den technokratischen Populismus in ein linkes oder rechtes Spektrum einzustufen, wodurch sich oft nichtpolitische Lösungen bieten, die in erster Linie auf der Unterscheidung zwischen "Gutem“ und „Bösem“ basieren (Bickerton/Accetti 20I8). Der technokratische Populismus verfolgt demnach das Ziel, Lagergrenzen aufzuweichen, da universell gültige Kategorien („gesunder Menschenverstand“, „tatsächlicher Wille des Volkes", worauf Carl Schmitt aufmerksam macht, usw.) angestrebt werden. Daher soll auch diese Perspektive im vorliegenden Aufsatz verfolgt werden.

\section{Die politische Bewegung ANO $2011 \mathrm{im}$ tschechischen Parteiensystem}

ANO 2OII wurde im Jahr 2OII als eine populistische und teils EU-skeptische politische Protestbewegung gegründet. Gründer von ANO ist Andrej Babiš, der bis heute an der Spitze dieser Bewegung steht und ein kontroverser Dollar-Milliardär sowie eine Symbolfigur der Oligarchisierung der tschechischen Politik ist. Nach der gewonnenen Wahl zum Abgeordnetenhaus 2017 hat er Anteile seiner Firmen in private Treuhandfonds abgetreten, um im Einklang mit der tschechischen Gesetzgebung zu handeln. ${ }^{2}$ Babiš bestimmt jedoch selbst die Mitglieder der Fonds und kann sie jederzeit abberufen.

Im Jahr 2017 umfasste sein Unternehmensimperium rund 250 Firmen in insgesamt I8 Ländern. Es handelt sich neben Chemiewerken, Geflügelmastanlagen, Fleischverarbeitungsbetrieben, Firmen für Landwirtschaftstechnik auch um Forstbetriebe (welt.de 2017). In Deutschland beschäftigt Babiš alleine in den Stickstoffwerken Piesteritz in Sachsen-Anhalt und in der Großbäckerei Lieken knapp 4500 MitarbeiterInnen. In der Tschechischen Republik gehört ihm außerdem seit 2013 zusätzlich das große Medienunternehmen Mafra, mit unter anderem zwei wichtigen Tageszeitungen, einem Rundfunksender, Druckereien und Internetportalen.

Der Wahlkampf der ANO-Bewegung wurde durch die US-amerikanische PR-Agentur Penn Schoen Berland unterstützt, die unter anderem bereits für Bill und Hillary Clinton tätig war. Als Ergebnis dieser Wahl entstand eine Koalition mit der ČSSD (Sozialdemokratische Partei) an der Spitze und ANO und KDU-ČSL (Christdemokratische Partei) als Koalitionspartner (Novotný/ Šárovec 2020).

2 Ein 2016 vom Parlament verabschiedetes Gesetz gegen Interessenkonflikte, das auch als „Lex Babiš“ bekannt ist, verbietet Regierungsmitgliedern inzwischen den Besitz von Medien. Tatsächlich hat Babiš seine Unternehmen danach formell einer Treuhandgesellschaft überschrieben, doch faktisch dürfte sein Einfluss kaum geschrumpft sein: Zentrale Rollen in der Treuhandgesellschaft spielen ein langjähriger Agrofert-Manager, ein Anwalt Babišs und die Ehefrau des Milliardärs 
Tabelle 1: Ergebnisse der Abgeordnetenhauswahl 2013

\begin{tabular}{lccc}
\hline & Stimmen (abs.) & Stimmen (in \%) & Sitze (abs.) \\
\hline ČSSD & 1016829 & 20,45 & 50 \\
ANO & 927240 & 18,65 & 47 \\
KSČM & 741044 & 14,91 & 33 \\
TOP 09 & 596357 & 11,99 & 26 \\
ODS & 384174 & 7,72 & 16 \\
ÚSvit & 342339 & 6,88 & 14 \\
KDU-ČSL & 336970 & 6,78 & 14 \\
\hline
\end{tabular}

Quelle: ČSÚ, Internet: https://volby.cz/pls/ps2013/ps2?xjazyk=CZ (Zugriff: 23/03/2020).

Tabelle 2: Ergebnisse der Abgeordnetenhauswahl 2017

\begin{tabular}{lccc}
\hline Partei & Stimmen (abs.) & Stimmen (in \%) & Sitze (abs.) \\
\hline ANO & 1500113 & 29,64 & 78 \\
ODS & 572948 & 11,32 & 25 \\
Piráti & 546393 & 10,79 & 22 \\
SPD & 538574 & 10,64 & 22 \\
KSČM & 393100 & 7,76 & 15 \\
ČSSD & 368347 & 7,27 & 15 \\
KDU-ČSL & 293643 & 5,80 & 10 \\
TOP 09 & 268811 & 5,31 & 7 \\
STAN & 262157 & 5,18 & 6 \\
\hline
\end{tabular}

Quelle: ČSÚ (2017), Internet: https://volby.cz/pls/ps2017nss/ps2?xjazyk=CZ (Zugriff: 23/03/2020).

Im Herbst 2017 ging Babiš als klarer Sieger aus den Wahlen zum Abgeordnetenhaus der Tschechischen Republik (Hanley/Vachudová 2018) hervor. Seine Protestbewegung ANO gewann mit dem deutlichen Vorsprung von 18 Prozentpunkten vor der zweitplatzierten ODS (Bürgerlich-Demokratische Partei). Doch mit Andrej Babiš wollten die meisten der etablierten Parteien keine Regierungskoalition bilden, da sie die Verbindung von Babiš zu seinem Unternehmen Agrofert, vor allem wegen der staatlichen Subventionen für die Landwirtschaft und dem auf ihm lastenden Verdacht der Zweckentfremdung von EU-Fördermitteln für äußerst problematisch empfanden. Im Juni 2018 bildete Babiš jedoch eine Koalition als Minderheitsregierung mit der Sozialdemokratischen Partei, welche auf die Unterstützung der Kommunistischen Partei angewiesen war.

Auch bei anderen Wahlen war die ANO 20II-Bewegung in Tschechien erfolgreich. So zum Beispiel wurde sie 2019 bei der Europawahl mit 21,18 Prozent die stärkste Kraft im Land. Bei den Kommunalwahlen 2014 erreichte ANO i4,6 Prozent. Auch bei den Kommunalwahlen 2018 etablierte sich ANO in den meisten größeren Städten als stimmenstärkste Kraft. Gesiegt hat ANO auch bei den Regionalwahlen 2016 (Šárovec 20I7; Pink/ Eibl 2018); hier erreichte sie in neun von dreizehn Regionen die meisten Stimmen. Insgesamt ist es ANO somit gelungen, ihre derzeit führende Stellung in der tschechischen Politik zu festigen.

Andrej Babiš setzt auf die romantisierte Vorstellung des „Volkes", das als eine mehr oder minder homogene Masse angesehen wird. Dies wird unter anderem an dem Kampagnenmotto von 2017 ersichtlich, welches besagt "Wir sind ein gutes Volk“. Bei etwa einem Drittel der WählerInnen in Tschechien kommt diese von professionellen PR-ExpertInnen angeordnete Argumentation offensichtlich gut an, da Babiš ein klares Bild von seinen typischen WählerInnen vor Augen hat: Sie stammen aus dem ländlichen Raum mit einem unterdurchschnittlichen Verdienst und haben keinen hohen Bildungsgrad. Deshalb orientiert sich seine Politik an diesen Schichten. Für Babiš sind das die „anständigen BürgerInnen“, die hart arbeiten. Ein Wahlkampf-Slogan von Babiš bei der Abgeordnetenhauswahl 2017 lautete: „Wir sind anders als die Politiker. Wir arbeiten." Die ANO-Bewegung kanalisiert Ängste und Befürchtungen, indem sie komplexe soziale und ökonomische Prozesse auf die vermeintlichen Verantwortlichen, die „untätigen PolitikerInnen“, reduziert. $\mathrm{Zu}$ den typischen technokratisch-populistischen Agitationstechniken der ANO-Bewegung gehört der Rückgriff auf die Common-Sense-Argumente. Hier bedient er sich der typischen populistischen Logik, welche die Gleichsetzung von individueller und kollektiver Moral nach dem Motto vertritt: Was sich im privaten 
Bereich bewährt und als richtig erwiesen hat, kann im öffentlichen Bereich nicht falsch sein.

\section{Methodik}

Die Daten, die hier analysiert werden, weisen fast immer eine Form räumlicher Autokorrelation oder räumlicher Abhängigkeit auf, da nahe beieinander liegende Orte eher dazu tendieren, ähnliche Eigenschaften zu haben, als Orte, die entfernt voneinander liegen(Tobler I970). Bei einer räumlichen Analyse oder einer Analyse der Dynamik der Veränderungen des WählerInnenverhaltens bieten sich für die Untersuchung gleich mehrere räumliche Techniken an. Hier wird die Analyse von räumlichen Ansammlungen der WählerInnenunterstützung mit Hilfe der Technik der räumlichen Autokorrelation verwendet, für deren Berechnung sich der Moran-Koeffizient eignet (Moran's I).

Werden die hohen Werte in einer Einheit von hohen Werten in benachbarten Einheiten begleitet (oder Orte mit niedrigen Werten, die miteinander benachbart sind), handelt es sich um eine positive räumliche Autokorrelation oder eine räumliche Ansammlung (spatial clustering). Sind Orte mit niedrigen Werten von Orten mit hohen umgeben (oder auch umgekehrt), handelt es sich um eine negative räumliche Autokorrelation, die es ermöglicht räumliche Abweichungen (spatial outliers) zu identifizieren und die Fälle der räumlichen Zufälligkeit der verfolgten Erscheinung zu repräsentieren (Fotheringham et al. 2002).

Vor der Berechnung der Indikatoren räumlicher Autokorrelation muss ein weiteres methodologisches Problem der räumlichen Analyse gelöst werden: Unterschiedliche Betrachtungen der Operationalisierung von räumlicher Nähe (aus Sicht der Ausgrenzung der benachbarten räumlichen Einheiten) können zu sehr differierenden Ergebnissen führen (vgl. Unwin/Unwin 1998). Die wichtigste Frage besteht in der Auswahl einer geeigneten Gewichtungsmatrix. Grundsätzlich gibt es zwei Grundtypen von Gewichtungsmatrizes: die diskrete sowie die verbundene. ${ }^{3}$ In unserem Fall wurde die räumliche Gewichtungsmatrix mit einer Grenzdistanz von Io Kilometern definiert. Ihre Eignung angesichts der Siedlungsstruktur der Tschechischen Republik wurde bereits durch erfolgreich durchgeführte Untersuchungen erwiesen (Bernard et al. 20I4).

Das Ergebnis der Messung von räumlicher Autokorrelation mit Hilfe des Moran-Koeffizienten ist ein Wert des statistischen Indikators, der das Ausmaß räumlicher Autokorrelation auf dem gesamten Gebiet identifiziert. Da unser Ziel die Analyse des WählerInnenverhaltens

3 Detaillierter zur Problematik der Auswahl der Gewichtungsmatrize vgl. Fotheringham et al. (2002: 42-45). auf kommunaler Ebene ist, um die potenziell unterschiedlichen Muster des WählerInnenverhaltens innerhalb der größeren Einheiten zu identifizieren, werden im ersten Schritt für die Identifizierung der räumlichen Ansammlungen ähnlicher Werte die Werte von räumlichen Autokorrelationen für jede analysierte Raumeinheit berechnet. Konkret wird der lokale Moran-Koeffizient (local indicators of spatial association, LISA) berechnet, der die Verteilung des globalen Moran-Koeffizienten ermöglicht.

Der lokale Moran-Koeffizient wird für jede Einheit extra berechnet; die statistisch erfassten Daten können als Karten dargestellt und in vier Gruppen kategorisiert werden (nach dem Typus der räumlichen Autokorrelation), welche den vier Quadranten des Moran-Diagrammes entsprechen (vgl. Abb. I). Auf diese Weise kann man die positiven oder negativen räumlichen Abhängigkeiten identifizieren, das heißt: die hohen Werte in einer Einheit mit ähnlichen Werten in einer anderen benachbarten Einheit mit hohen Werten (hot spots) oder die auf der anderen Seite niedrigen Werte von Variablen mit ähnlichen niedrigen Werten in benachbarten Einheiten (cold spots). Auch gilt dies für räumliche Abweichungen (spatial outliers), d.h. hohe Werte, die umgeben sind von niedrigen Werten und vice versa (vgl. Anselin 1995). In diesem Aufsatz werden die Karten benutzt, um die Werte von allen vier Quadranten des Moran-Diagrammes darzustellen, wobei die farbige Darstellung der Karten des Indikatoren LISA den Farbtönen in Abbildung I entspricht.

\section{Abbildung 1: Moran Diagramm}

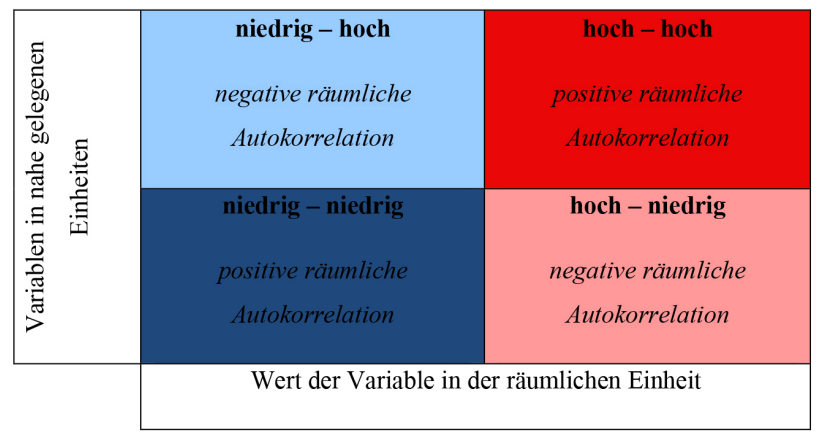

Quelle: Autoren

Unser Ziel ist nicht nur die Beschreibung der räumlichen Ansammlung der WählerInnenunterstützung während einer Wahl, sondern auch die Analyse der Ansammlung der Muster der Parteienunterstützung zwischen den einzelnen Wahlen. Dabei wird neben der univariaten (eindimensionalen) Version des lokalen Moran-Koeffizienten, der die räumliche Ansammlung der WählerInnenunterstützung während einer Wahl misst, auch die bivariate (zweidimensionale) Variante des lokalen Moran-Koeffizienten verwendet. Sie ermöglicht die 
Analyse der raumzeitlichen (Un-)Stabilität (spatiotemporal non-stability) der Unterstützung einer Partei bei zwei Wahlen sowie die Untersuchung der geographischen Verschiebungen der Stimmen zwischen Parteien bei zwei aufeinander folgenden Wahlen (vgl. Anselin 1995, I55-I60; Shin/Agnew 2007, 294). Mit Rücksicht auf den räumlichen Charakter der geographischen Daten kann der zweidimensionale lokale Moran-Koeffizient der Karten aufzeigen, dass räumliche Muster versteckte (underlying) Prozesse und Akteure indizieren, die sich in einer bestimmten Zeit bilden und modifizieren (Fortin/ Dale 2009). Sie können deshalb darstellen, wie der Prozess der (Un-)Stabilität und der Veränderung des WählerInnenverhaltens geographisch bedingt ist, konkret im Fall einer schrittweisen oder sofortigen Veränderung der WählerInnenunterstützung zwischen den einzelnen Wahlen (Shin/Agnew 2007).

In diesem Aufsatz konzentrieren wir uns auf die Wahlen zum Abgeordnetenhaus in den Jahren von 2013 bis 20I7.4 Die ANO-Bewegung besiegte zwar bei der Wahl im Jahr 2013 die ODS, landete jedoch im Gesamtergebnis auf dem zweiten Platz hinter der Sozialdemokratischen Partei. Nach vier Jahren siegte ANO schon deutlicher, da sie nun in den traditionell ČSSD geprägten Regionen stärker vertreten war. Da der Parteienwettbewerb zwischen der ODS als stärkste Partei im politischen Spektrum rechts der Mitte und der ČSSD als stärkste Partei links der Mitte bei der Wahl zum Abgeordnetenhaus 2006 am deutlichsten war (vgl. Balík/ Hloušek 20I6), zeigen wir am Beispiel dieser Wahl die Erfolge dieser Parteien auf den Karten, um das anschließend mit den Verschiebungen zu Gunsten der ANOBewegung während der Wahlen von 2013 und $2017 \mathrm{zu}$ vergleichen. Die Wahl von 2006 zeigt des Weiteren die regionalen Unterschiede zwischen der Unterstützung der beiden ehemaligen großen Parteien in Tschechien auf. Beiden ist es bei dieser Wahl gelungen, zusammen insgesamt 67,07 Prozent der WählerInnenstimmen zu erhalten. An der Kartendarstellung im nächsten Kapitel wird ersichtlich, dass die ANO-Bewegung sowohl in den traditionellen ODS-starken als auch ČSSD-starken Regionen erfolgreich war. Dadurch ist es ihr gelungen, aus beiden Parteien Kleinparteien zu machen und damit zur Neugestaltung des Parteiensystems beizutragen.

4 Wir verwenden die statistischen Daten von Wahlen zum Abgeordnetenhaus von 2006 bis 2017 auf der Ebene der mehr als 6000 tschechischen Städte und Gemeinden. Doch die Karten enthalten nicht die administrativen Grenzen dieser Städte und Gemeinden, sondern nur den Farbton bei der Identifizierung einer positiven räumlichen Autokorrelation von hohen oder niedrigen WählerInnenunterstützungswerten. Die Karten werden jedoch zur besseren Veranschaulichung durch Grenzen der tschechischen Regionen ergänzt.

\section{Die Wahlgeographie der Tschechischen Republik}

Wird auf die letzten 30 Jahre zurückgeblickt, gehört das tschechische Parteiensystem zu den stabilsten und konsolidiertesten Parteiensystemen in den Ländern Mittel- und Osteuropas. Nach dem Fall des kommunistischen Regimes und der Einführung des freien Parteienwettbewerbs $1990 \mathrm{kam}$ es zu einer raschen Entstehung der politischen Parteien. Schnell gewann vor allem die sozioökonomische cleavage an Bedeutung, nach der sich das Parteiensystem orientierte (Hloušek/Kopeček 2008). Untersuchungen von Politikwissenschaftlerinnen und Politikwissenschaftlern bestätigten die Verbindung zwischen unterschiedlichen sozialen Gruppen und politischen Parteien bei den Wahlen von I990 bis 20I0. Die Untersuchung von Linek und Lyons (2013), die sich mit dem Zeitraum von 1990 bis 2010 beschäftigten, zeigt, dass die Wahlpräferenz in Tschechien vorwiegend auf drei sozioökonomischen und demographischen Merkmalen basierte: die soziale Klasse, die Religion und das Alter. Die politischen Parteien ließen sich anhand klarer ideologischer Orientierung relativ stabil in die LinksRechts-Skala einordnen. Während die bürgerlich-demokratische Partei (ODS) vorwiegend von liberalen und konservativen WählerInnen, die aus höheren Schichten stammten, gewählt wurde und damit bis 2013 die dominante Partei im rechten politischen Spektrum war, war die Sozialdemokratie (ČSSD) politisch links der Mitte am erfolgreichsten. Daneben etablierte sich die KSČM als die dritte Kraft in den peripheren Gebieten, als Alternative für ArbeiterInnen und die ältere Generation (Novotný 2017).

Die vierte politische Partei im tschechischen Parteienspektrum ist die der Christdemokraten (KDU-ČSL), die mehrheitlich von KatholikInnen gewählt wird. Für diese WählerInnen ist gerade die Religion die wichtigste cleavage. Die KDU-ČSL bewegt sich an der Fünf-ProzentHürde und fungierte in den Jahren von 1990 bis 2010 als wichtiges Zünglein an der Waage bei der Regierungsbildung. Gerade ihre Abgeordneten waren im tschechischen politischen System, in dem Koalitionen von drei Parteien entstehen mussten, entscheidend (vgl. Linek/ Lyons 20I3). Bis 2013 gab es außerdem im Abgeordnetenhaus immer eine weitere Partei, die meist Mitterechts platziert war; diese hieß bis 1998 Bürgerlich-demokratische Allianz (ODA), danach Freiheitsunion (US) oder Öffentliche Angelegenheiten (VV).

Erkenntnisse von WahlgeographInnen haben bestätigt, dass die kontextuellen Variablen, wie die soziale, politische oder wirtschaftliche Situation bei den Wahlen von 1990 bis 2010 entscheidend für das Wahlverhalten war (Kostelecký/Bernand 20I4). Dies führte zu einer recht stabilen Unterstützung der Parteien, die die ersten zwanzig Jahre mit wenigen Ausnahmen bestehen blieb (Bernard/Šimon 20I4; Maškarinec 20I7). Die liberalen 
und konservativen Parteien hatten damit mehr WählerInnen in den wirtschaftlich stärkeren Regionen, während die sozialdemokratischen sowie kommunistischen Parteien dagegen ihre AnhängerInnen meist in strukturell schwachen Gebieten verorten konnten. Diese klare Unterscheidung wurde auch bei den Präsidentschaftswahlen von 2013 (Wahlkampf Zeman-Schwarzenberg) und 2018 (Wahlkampf Zeman-Drahoš) deutlich (Maškarinec 2013; Novotný 2015; Sax 2018).

Die konservativen sowie liberalen Parteien konnten ihre besten Ergebnisse in der Hauptstadt Prag oder in den Regionen Pilsen, Budweis, Liberec oder Hradec Králové verzeichnen. In Mähren waren diese Parteien vor allem in den großen Städten Brünn und Ostrava erfolgreich. Die christdemokratische Partei dagegen hatte und hat bis heute ihre meisten WählerInnen in Südmähren und in den ländlichen Regionen Süd- und Ostböhmens, da hier bis heute die meisten Gläubigen leben, wobei mehrheitlich die KatholikInnen diese Partei wählen. Dies wird auch in den Karten ersichtlich, da die Christdemokraten fast ausschließlich in Mähren, also im östlichen Teil des Landes, erfolgreich waren. In den peripheren Grenzgebieten sowie in den ehemaligen Sudetengebieten unterstützen die WählerInnen dagegen hauptsächlich die kommunistische Partei (Bernard/ Šimon 20I4; Bernard et al. 20I4; Maškarinec 2017). demokratie. Dagegen hatte die ČSSD in großen böhmischen Städten oder allgemein gesprochen in Böhmen deutlich weniger SympathisantInnen, da hier die ODS das politische Geschehen dominierte. Eine hohe Unterstützung der KSČM für die Zeit von I99o bis 20Io konnte in drei Gebieten beobachtet werden. Während das wichtigste Zentrum die Grenzgebiete in Nord- und Westböhmen waren (Novotný 2017), konnte sich die Partei auf starke Wählerschaften auch in den ländlichen Gebieten Süd- und Nordmährens verlassen. Bis heute sind die drei stärksten Gebiete der KSČM-WählerInnen die Peripherien, welche bis 1945 mehrheitlich von deutschsprachiger Bevölkerung bewohnt wurden. Die ČSSD und KSČM hatten zudem bis 2010 ihre SympathisantInnen hauptsächlich in den Peripherien im Landesinneren, sprich außerhalb der großen Städte, aber auch in den ländlichen Regionen mit Menschen mit unterdurchschnittlichem Verdienst oder Bildungsgrad (Bernard/ Šimon 20I7).

\section{Die Wahlerfolge der ANO 2011-Bewegung}

Während die Wählerschaft der traditionellen tschechischen Parteien in den ersten zwanzig Jahren seit der politischen Wende in klar abgegrenzten Regionen

Abbildung 2: Lokale räumliche Autokorrelation (Cluster) - Karten mit den Wahlerfolgen von ODS und KDU-ČSL, 2006
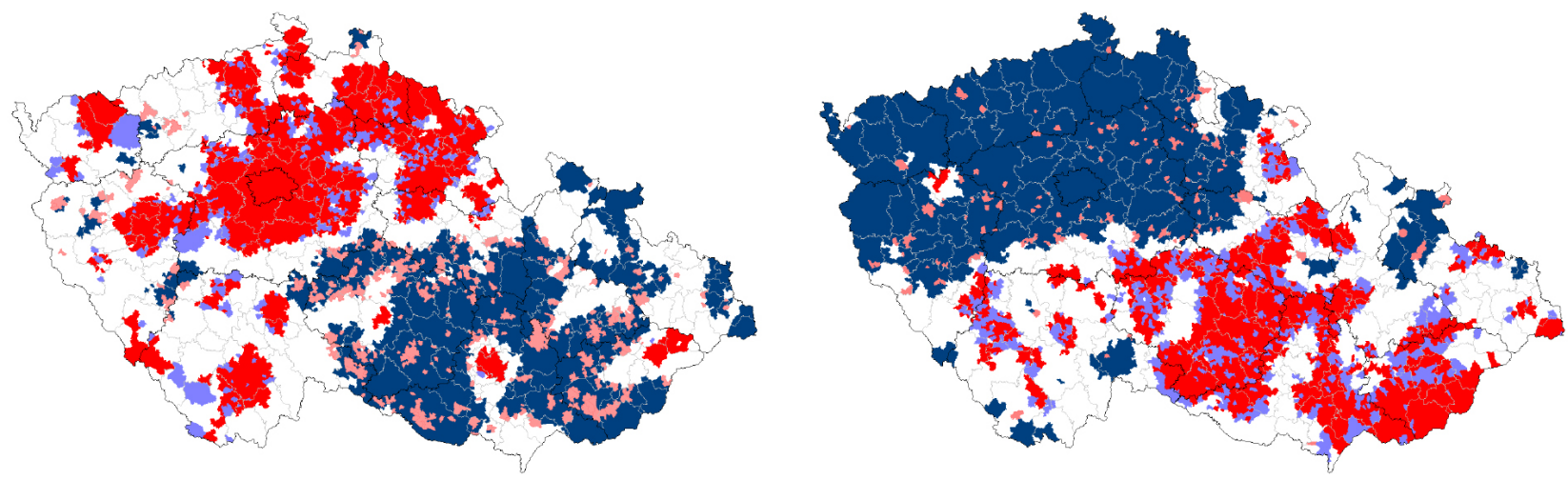

Quelle: eigene Darstellung, Čsú, Internet: https://volby.cz/pls/ps2013/ps2?xjazyk=CZ (Zugriff: 23/03/2020).

Allgemein gesprochen gilt, dass die Sozialdemokratie und die Kommunistische Partei Böhmens und Mährens zwischen 1990 und 20IO, aber teilweise auch später, hauptsächlich in den strukturell schwachen Teilen Tschechiens stark waren. Auch hier kann anhand der Karten überprüft werden, dass die Hochburg der ČSSD in Zentral- und Nordmähren lokalisierbar war (hauptsächlich in der Städteagglomeration um Ostrava, Karviná und Frýdek-Místek). Vor allem in der Zeit der schrittweisen Stilllegungen der Kohlegruben um die Jahrtausendwende entstand dort ein tiefes Gefühl der Verbundenheit der BewohnerInnen mit der Sozial- konzentriert war, sind solche Aussagen über die Wahlunterstützung der ANO-Bewegung nicht machbar. Die Partei von Andrej Babiš kann somit aus Sicht der Wahlgeographie nicht klar eingeordnet werden.

Bei der Wahl zum Abgeordnetenhaus von 20I3, bei der die ANO-Bewegung zum ersten Mal kandidierte, stammten die meisten WählerInnen fast ausschließlich aus Böhmen und Mittelmähren. Doch bereits zu dieser Zeit war es nicht mehr möglich, den typischen Wähler bzw. die typische Wählerin mit einer konkreten ideologischen Präferenz zu identifizieren. Im Gegenteil gehörten zu den UnterstützerInnen sowohl Liberale als auch 

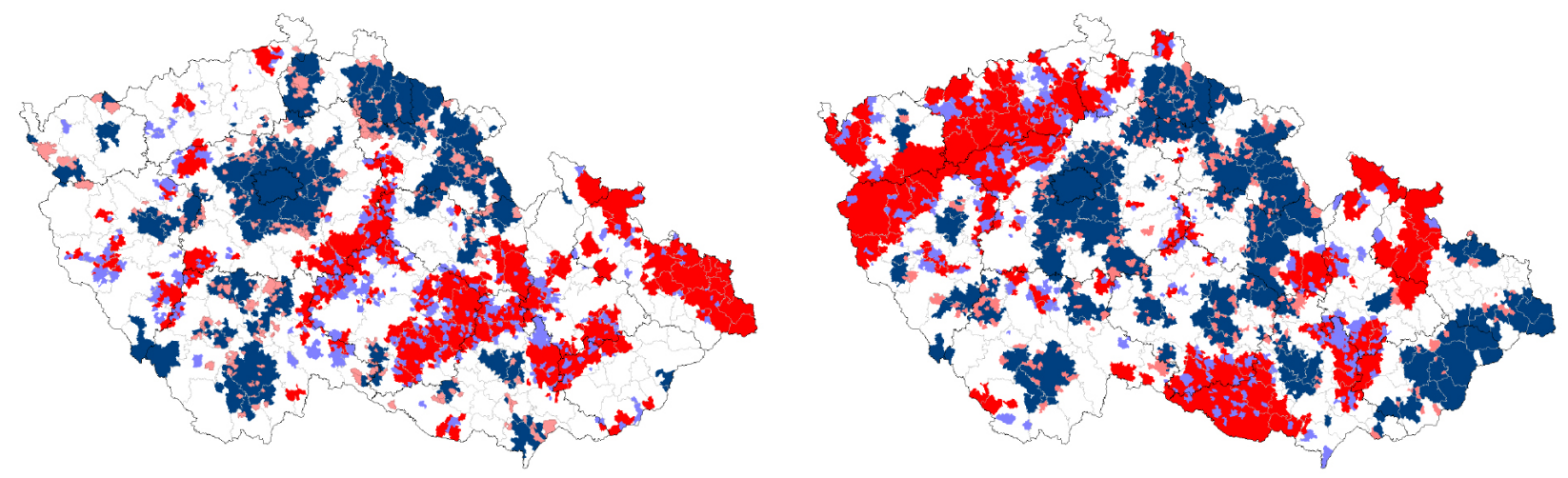

Quelle: eigene Darstellung, Čsú, Internet: https://volby.cz/pls/ps2013/ps2?xjazyk=CZ (Zugriff: 23/03/2020).

Konservative, besonders die vormaligen ODS-WählerInnen, wie auch SozialdemokratInnen oder Angehörige der Linken. Es war die Folge der Entideologisierung des Programms der ANO-Bewegung, das nicht eindeutig einer ideologischen Ausrichtung zugerechnet werden konnte. Doch wird die Karte der Wahlerfolge der ANOBewegung bei der Wahl zum Abgeordnetenhaus 2013 näher betrachtet, fällt auf, dass die Regionen des Wahlerfolges von ANO etwa gleichzusetzen sind mit den früheren Hochburgen der ODS bis 20IO. Diese dominante rechtsliberale und konservative Partei siegte bis dahin vorwiegend in Prag und Mittelböhmen und in großen böhmischen Städten wie Budweis, Pilsen oder Hradec Králové. Babiš fokussierte mit seiner Wahlkampagne 2013 hauptsächlich diese Wählerschaft, da sie mit den Korruptionsskandalen der Parteispitze um Petr Nečas, welche stark medialisiert wurden, besonders unzufrieden waren. Des Weiteren ist an dieser Stelle anzumerken, dass die Wahl in einer brisanten Zeit durchgeführt wurde, da hier die traditionellen Parteien, allen voran die ODS, zerstritten waren und durch die Affären um Nečas ihr Zerfall begünstigt wurde. Demnach waren es keine spezifischen programmatischen Punkte, die ANO von der ODS übernommen hatte. Vielmehr entschieden die unzufriedenen WählerInnen aufgrund der Umstände und der Krise der ODS, der ANO-Bewegung ihre Stimmen zu geben, da ANO für viele eine Alternative darstellte. ANO wurde als eine Antikorruptionsbewegung gesehen, mit der sich die WählerInnen identifizieren konnten. Andrej Babiš schaffte es so, die ODS, die traditionell stärkste Partei rechts der politischen Mitte, abzulösen.

Doch bereits bei der Wahl im Jahr 2013 fiel auf, dass Andrej Babiš nicht nur in den Regionen Tschechiens mit der vormals starken Unterstützung der ODS, sondern auch in anderen Regionen Erfolge verbuchen konnte, die traditionell eher links geprägt waren. Neue WählerInnen erhielt ANO in Regionen, in denen die Kommunisten stark vertreten waren, vor allem in den periphe- ren Gebieten in Nordwestböhmen. Die ANO-Bewegung ordnete sich gleich mit ihrem ersten Erfolg nicht auf der traditionellen Rechts-Links-Skala der politischen Parteien ein, denn es zeigte sich, dass die professionelle Wahlkampagne und die Betonung des neuen politischen Stils auf beiden Seiten des politischen Spektrums WählerInnen ansprechen konnte. Mit den neuen Themen, vor allem mit dem Fokus auf den Kampf gegen die Korruption und mit dem populistischen kommunikativen Stil konnte sie WählerInnen sowohl aus dem rechten als auch dem linken politischen Spektrum ansprechen.

Bei der Wahl 2017 zum Abgeordnetenhaus siegte ANO zum ersten Mal und dies mit deutlichem Vorsprung. Die Wahlergebnisse lieferten zudem eine Neuverteilung der regionalen Unterstützung. Während die ANO-Bewegung bei der Wahl 2013 vor allem in den Gebieten erfolgreich war, in denen bis 2010 die liberal-konservative ODS ihre meisten SympathisantInnen hatte, sprach sie bei der Wahl von 20I7 vor allem die WählerInnen in den traditionell sozialdemokratischen Regionen an. Der Zuwachs der Unterstützung der ANO-Bewegung wurde auf Grund der sinkenden Wählerschaft der beiden linken Parteien, der ČSSD und KSČM, möglich. ANO 2OII siegte somit in den nördlichen Grenzgebieten und in den strukturell schwachen Gebieten, die bis zu dieser Wahl überwiegend sozialdemokratisch oder kommunistisch waren. Das gilt auch für den südlichsten Teil Mährens (Region um Znojmo). Im Vergleich zu 2013 sank der Erfolg von ANO in Prag und in der Prager Agglomeration deutlich.

Wie in Abbildung 4 (linke Karte) zu sehen ist, kollidierte die Unterstützung der ANO-Bewegung bei der Wahl von 2013 mit den Wahlerfolgen der Parteien rechts der politischen Mitte, vor allem der ODS. Wir sehen große cluster mit einer großen prozentualen Verteilung zugunsten der ANO-Bewegung in den traditionell starken Regionen der ODS. Das galt für Prag und Brünn, aber auch für andere größere Städte vor allem in Böhmen. Dort brach zu dieser Zeit das Vertrauen der ansonsten 
Abbildung 4: Lokale räumliche Autokorrelation (Cluster) - Karten mit den Wahlerfolgen von ANO, 2013/2017
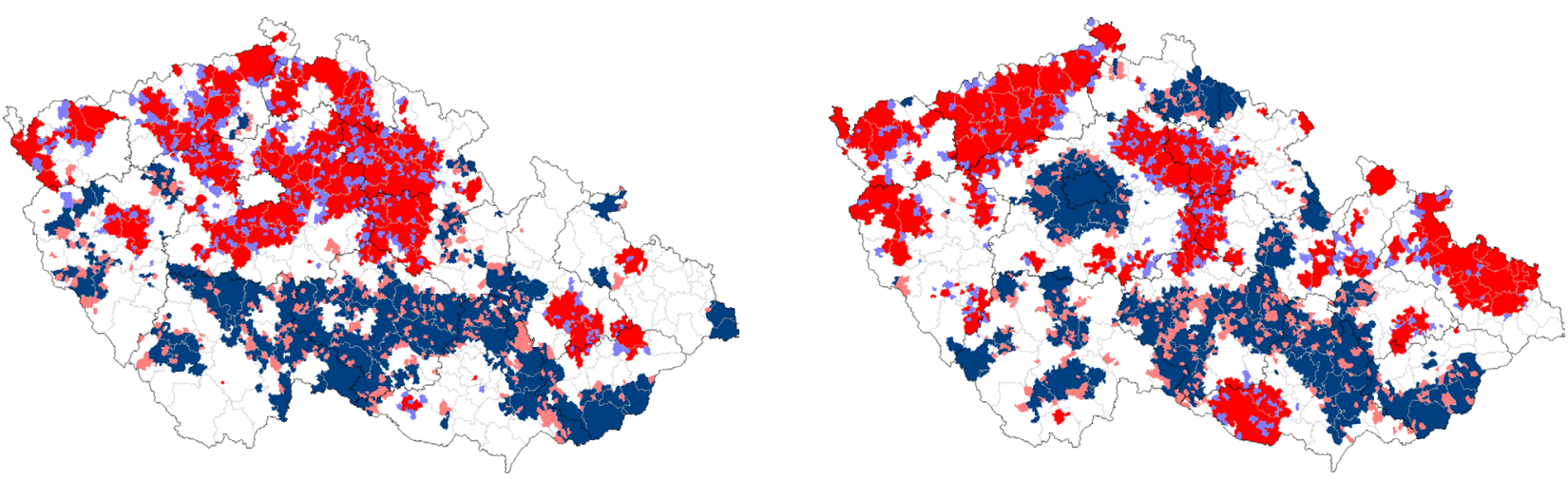

Quelle: eigene Darstellung, Čsú, Internet: https://volby.cz/pls/ps2013/ps2?xjazyk=CZ (Zugriff: 23/03/2020).

sehr disziplinierten WählerInnen zur ODS weg und es fand der Übergang zur Unterstützung der ANO-Bewegung statt. Daneben war bereits bei der Wahl von 2013 ANO in Bezirken wie Ústí nad Labem und Karlsbad im Nordwesten des Landes erfolgreich, wo traditionell eher die sozialdemokratischen und kommunistischen Parteien erfolgreich waren. Schon im Jahr 2013 wurde ersichtlich, dass ANO 2OII auf Grund der Entideologisierung und des Populismus WählerInnen vom linken und rechten Spektrum ansprechen konnte, auch wenn die wichtigsten Zugewinne durch die Verluste der ODS an die ANO-Bewegung zu erklären sind.

Sehr unterschiedliche Muster fallen im Vergleich der Unterstützung der ANO-Bewegung von 2017 und der Erfolge der Parteien links der Mitte (ČSSD und KSČM) auf. In diesem Fall zeigt die Karte eine fast komplette Veränderung des ANO-Elektorats bei den zwei aufeinander folgenden Wahlen. Wir sehen große und kompakte cluster mit hoher Übereinstimmung zwischen der ANO-Bewegung und der ČSSD und KSČM, vor allem im Nordwesten des Landes (im Erzgebirge und im Norden Mährens, wo früher die Hochburg der Sozialdemokratie war).

Die geographische Untersuchung der Wahlerfolge der ANO-Bewegung im Zuge der Wahlen zum Abgeordnetenhaus zwischen 2013 und 2017 zeigt, wie unterschiedlich die Bewegung von Seiten der WählerInnen wahrgenommen wurde. Aus der Analyse geht hervor, dass der ursprüngliche Erfolg mit der Übernahme von vor allem rechtsliberalen WählerInnen in Mittel- und Ostböhmen bedingt war und dass es Babiš zugleich gelungen ist, neue Wählergruppen in traditionell sozialdemokratisch und kommunistisch geprägten Regionen Nordwestböhmens zu gewinnen. Dagegen etablierten sich die WählerInnen der ANO-Bewegung bei der Abgeordnetenhauswahl aus dem Jahr 2017 fast ausschließlich aus dem traditionellen sozialdemokratischen und kommunistischen Milieu.

Die Transformation der ANO-WählerInnen zwischen 2013 und 2017 hatte auch weitere Folgen. So zum Beispiel bezeichneten einige tschechische

Abbildung 5: Lokale räumliche Autokorrelation (Cluster). Karten der Verteilung der Unterstützung für die ANO 2011 mit rechten und linken Parteien

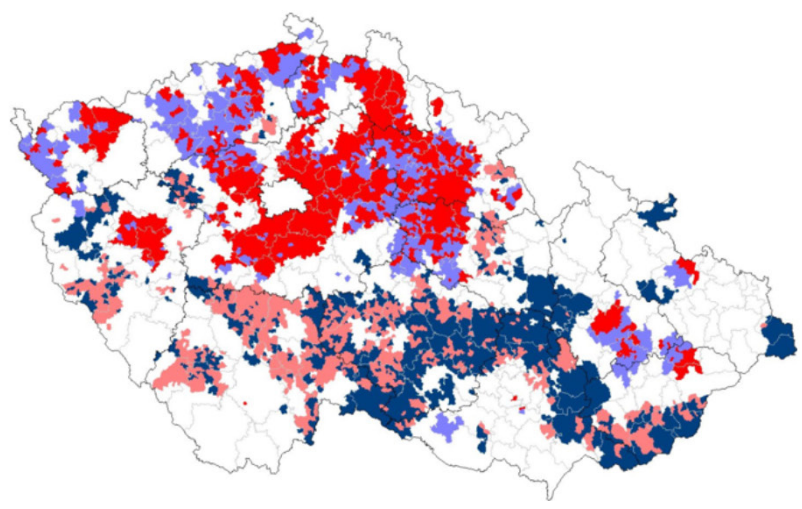

RECHTE PARTEIEN (2006) vs. ANO (2013)

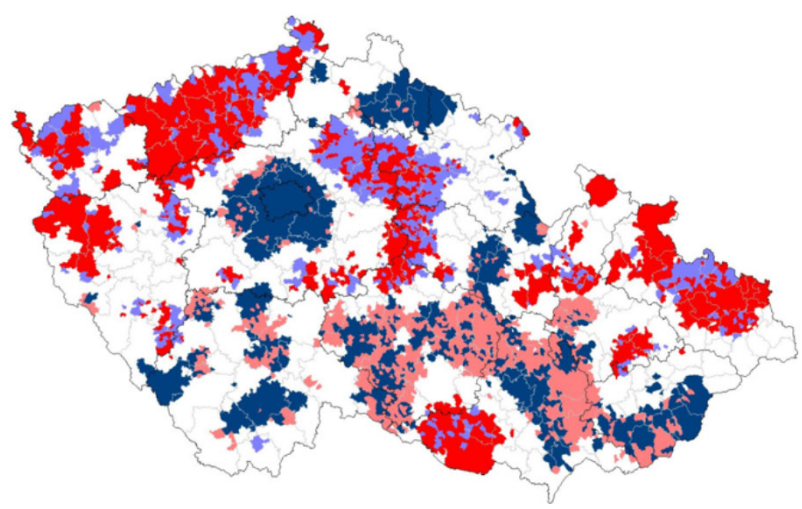

LINKE PARTEIEN (2006) vs. ANO (2017)

Quelle: eigene Darstellung, Čsú, Internet: https://volby.cz/pls/ps2013/ps2?xjazyk=CZ (Zugriff: 23/03/2020). 
PolitikwissenschaftlerInnen (etwa Havlík/Voda 20I6) die ANO-Bewegung nach der Wahl 2013 als dominante Partei rechts der Mitte im tschechischen Parteiensystem. Durch den Erfolg von 2013 bei den rechtsliberalen WählerInnen beendete die ANO-Bewegung ihre Agitation zu Gunsten dieser Wählerschaft; sie hörte auf, sich gegen die alten Parteien zu stellen und konzentrierte sich primär auf die sozialdemokratischen und linken WählerInnen. Die Partei konnte auf Grund ihres PRTeams die Erfolge der gemeinsamen Regierung mit den Sozialdemokraten besser verkaufen. Bei der Wahl von 2017 wurde sie deshalb für die nicht stabilen WählerInnen der Sozialdemokratie glaubwürdiger als die ČSSD selbst.

Havlík und Voda (20I8) bieten dafür weitere Erklärungsansätze, etwa dass die Wahl von 2017 gezeigt hat, dass für die tschechischen WählerInnen nicht mehr die Zugehörigkeit zu einer sozialen Gruppe als Faktor der Parteipräferenz bedeutend ist, sondern dass die Merkmale der Postpolitik stärker berücksichtigt werden (s. Mouffe 2005). Die Rechts-Links-Skala spielte nun bei dieser Wahl nicht mehr die wichtigste Rolle. Die früheren großen Parteien, also die ODS und ČSSD, verloren beide ihre dominante Stellung. Entscheidend für die tschechischen WählerInnen ist nicht mehr die Ideologie oder das Programm, sondern eine gute Wahlkampagne und die Fähigkeit, Interesse zu wecken sowie neue Sichtweisen auf die Politik zu liefern. Beides kann die ANOBewegung sehr gut, unter anderem auch auf Grund des technokratischen Populismus, durch den es Babiš gelingt, die Probleme der Bevölkerung vor allem in den ländlichen Regionen wahrzunehmen und in eine gezielte Agitation umzuwandeln.

Aus der Wahlanalyse geht hervor, dass sich die geographische Verteilung der Unterstützung der ANO-Bewegung zwischen den Wahlen von 2013 und 2017 deutlich verändert hat. Im Jahr 2013 war die Partei auf Grund ihrer starken Anti-Korruptions-Agitation vor allem in den traditionellen ODS-starken Gebieten erfolgreich, da sich die seit I99I bestehende ODS zu dieser Zeit in einer Krise mit vielen Korruptionsskandalen befand, sodass die Wählerunterstützung rasant gesunken ist. Die vier Jahre in der Opposition halfen jedoch der ODS, sich von der bisherigen Führung zu trennen und einen Neuanfang zu wagen.

Vier Jahre gemeinsame Koalition zwischen der ANOBewegung und der Sozialdemokratie mit dem Ministerpräsidenten Bohuslav Sobotka und den Christdemokraten bedeuteten letztlich den Sieg der ANO-Bewegung bei der Wahl 2017 - in Verbindung mit deutlichen Verlusten für die beiden Koalitionspartner. Es veränderten sich die programmatischen Akzente von Babiš, der mit seinen PR-ExpertInnen die öffentliche Meinung und die Gedankenströmungen in der Bevölkerung beobachtete und auswertete. Die Verschiebung der Wahlkampfthemen in
Richtung links bedeutete zusammen mit einer personellen und ideologischen Krise beider Parteien nach der Wahl 20I7, dass sich die traditionellen WählerInnen der Sozialdemokratie und der Kommunistischen Partei eher mit Babiš identifizierten.

Angesichts dieser Ergebnisse, die aus den Karten ersichtlich werden, stellt sich die Frage, inwieweit in Tschechien der Weg schrittweise zurück zu einem LinksRechts-Parteienwettbewerb bestritten werden kann, wenn die Unterstützung der ANO-Bewegung nun zu Ungunsten der linken Parteien zunimmt. Zudem lässt sich schwierig einschätzen, welche der aktuell neun im Parlament vertretenen Parteien eine dominante Stellung rechts der Mitte einnehmen wird. Am ehesten wäre dies die ODS, die seit der Wahl 2017 wieder Stimmengewinne verzeichnen konnte. Außerdem kann Babiš mit seinem professionellen Team die nächsten Kampagnen wieder thematisch umdrehen und neue Wählergruppen gewinnen.

\section{Fazit}

Überschreitet die räumliche Unterstützung der ANO-Bewegung angesichts ihrer programmatischen Entideologisierung und ihres technokratischkommunikativen Stils die langfristigen räumlichen Trennlinien des tschechischen Parteienspektrums oder ist sie vorzugsweise in traditionell linken oder rechten Gebieten der Tschechischen Republik erfolgreicher, übernimmt also nur WählerInnen aus den traditionellen Blöcken des tschechischen Parteienspektrums? Und was ist die programmatische Besonderheit des technokratischen Populismus der ANO-Bewegung angesichts der Wahlergebnisse von 2013 und 20I7? Dieser Aufsatz suchte Antworten auf diese zwei Forschungsfragen, zum einen mit Hilfe von wahlgeographischen Techniken, zum anderen in Konfrontation mit der Theorie des technokratischen Populismus, mit dem wir zeigen wollten, inwiefern Babiš programmatisch flexibel handeln kann.

Zur ersten Forschungsfrage: Die ANO-Bewegung erhielt in beiden untersuchten Wahlen zum Abgeordnetenhaus ihre Stimmen vor allem in den Regionen mit unzufriedenen WählerInnen. In beiden Fällen kann also über das Phänomen einer Protestwahl gesprochen werden. Lediglich die Gründe waren von unterschiedlicher Natur. Bei der Wahl 20I3 waren einige BürgerInnen auf kommunaler sowie nationaler Regierungsebene unzufrieden mit der Art des Regierens der ODS. Dies wurde vor allem in Prag deutlich. Babiš konzentrierte sich deshalb auf diese enttäuschten BürgerInnen und bot ihnen mit Hilfe professioneller politischer Kommunikation ein Antikorruptionsprogramm an. Die Karten bestätigen, dass sich die Wahlunterstützung von ANO mit den 
traditionell starken Gebieten der ODS-WählerInnen überschnitt. Diese WählerInnen unterstützten Babiš aus Protest, und bald zeigte sich, dass sie schrittweise zur ODS zurückkehren wollten. Babiš erkannte zudem in der gemeinsamen Regierung mit der Sozialdemokratie, dass es auch links der Mitte unzufriedene Wählergruppen gibt.

Zur zweiten Forschungsfrage: Die ANO-Bewegung war von Beginn an anders als die kleinen Parteien, die immer nur eine oder zwei Wahlperioden überstanden. Andrej Babiš betrat die politische Bühne ausgestattet mit viel Erfahrung, Kapital und erfahrenen PR-ExpertInnen. Er tritt als technokratischer Populist auf und bedient sich der bereits bekannten Kommunikationsstilelemente populistischer Parteiführer wie den Antagonismus zwischen Eliten („die da oben“) und Volk („wir hier unten“), den Appell an die „Verlierer“, den Rückgriff auf die Common-Sense-Argumente sowie die Vorliebe für radikale Lösungen. Doch was ihn von anderen unterscheidet, betrifft die Umsetzung dieser Elemente in die Praxis. Zwar ist er antagonistisch gegenüber den Eliten, doch muss er das allein deshalb anders machen, weil er schon seit mehr als sechs Jahren in der Regierung sitzt, mittlerweile als Ministerpräsident. Außerdem ist er nicht nur ein Populist, sondern er ist vielmehr ein technokratischer Populist. Es finden sich in seinem Umkreis genug ExpertInnen, die wichtige Funktionen in seiner Regierung einnehmen. Mitgliedschaft in seiner ANOBewegung spielt dabei eine minimale Rolle. Er hat insgesamt 78 von 200 Mitgliedern im Abgeordnetenhaus und mit ihrer Hilfe kann er seine Regierungsvorhaben umsetzen.

Sehr deutlich war die Veränderung der Wählerunterstützung von ANO 201 I aus Sicht der regionalen Verteilung der Wählerschaft. Bei der Wahl zum Abgeordnetenhaus von 2013 war die ANO-Bewegung vor allem in denjenigen Regionen und Gemeinden erfolgreich, in denen traditionell die Parteien rechts der Mitte ihre WählerInnen angesprochen haben. Durch seine Wahlkampagne schaffte er es, gegen die Korruptionsskandale der ODS zu arbeiten. Obgleich die Negativkampagne gegen die ODS noch nicht untersucht wurde, kann wohl angenommen werden, dass sie erheblichen Anteil am schlechtesten Ergebnis der ODS in deren Parteigeschichte hatte. Doch daneben erzielte bereits bei dieser Wahl von 2013 die Partei von Babiš Erfolge auch in einigen Wahlbezirken, in denen bisher die Parteien links der Mitte (ČSSD und KSČM) dominiert hatten. Bei der letzten Abgeordnetenauswahl $2017 \mathrm{kam}$ es jedoch zu einer deutlichen Wende. Durch Kartendarstellungen konnte in dieser Untersuchung dargelegt werden, dass sich die Unterstützung für die ANO-Bewegung in Regionen verschoben hatte, in denen die WählerInnen bisher vor allem die ČSSD und KSČM wählten. Begründet werden könnte dies mit der koalierenden Regierung zwi- schen der ČSSD, ANO und der KDU-ČSL, in der Bohuslav Sobotka als Premier agierte. Andrej Babiš schaffte es hier immer wieder, die Erfolge der Regierung als seine eigenen darzustellen. Zusätzlich kamen auch persönliche Streitigkeiten zwischen Sobotka und Babiš hinzu, in denen Sobotka beispielsweise dem Vorsitzenden des Koalitionspartners ANO vorhielt, seine privaten Einkünfte nicht ausreichend offen zu legen. Im Mai 2017 schaffte Sobotka es, den Rücktritt von Babiš von seinen Positionen als Vizepremier sowie Finanzminister durchzusetzen. Dies trug sicherlich zu den konfliktlastigen Auseinandersetzungen der beiden Parteien in den Wahlkampagnen von 2017 bei.

Diese Veränderungen im Wahlverhalten in einer so kurzen Zeit hat die Tschechische Republik in den letzten 30 Jahren nicht erlebt. Die fast vollständige Veränderung der Wählergruppen der ANO-Bewegung zwischen 2013 und 2017 deutet darauf hin, dass die Erklärkraft der Rechts-Links-Skala und die/der Ideologien in Hinblick auf das Wahlverhalten in Tschechien tendenziell eher sinkt. Die programmatische Entideologisierung der ANO-Bewegung gibt in Kombination mit dem technokratischen Populismus des Parteiführers Andrej Babiš Antworten darauf, warum es gerade der ANOBewegung gelingt, innerhalb von einer Wahlperiode für so unterschiedliche Wählergruppen interessant zu sein.

Gleichzeitig müssen wir aber die Annahme ablehnen, dass als Folge der programmatischen Entideologisierung und des technokratischen kommunikativen Stils der ANO-Bewegung die Wählerunterstützung in Tschechien überall konstant ist. Denn gerade eine Tatsache zeigt, dass eine ideologische „Flüssigkeit" es Babiš ermöglicht, flexibel auf die Veränderung von Stimmungen in der Gesellschaft zu reagieren. Und zwar, dass der Erfolg der ANO-Bewegung 2013 größtenteils auf seiner Anziehungskraft für Mitte-Rechts-WählerInnen in Regionen mit einer spezifischen Verteilung bestimmter sozioökonomischer Gruppen (ArbeitgeberInnen, Selbstständige, städtische Intelligenz) oder einer regionalen Kultur, die historisch gesehen Unternehmertum und Unabhängigkeit betonte, ruhte, und sich 2017 grundlegend veränderte, als die ANO-Bewegung vor allem in fast ausschließlich linksgerichteten Gebieten in strukturell benachteiligten Regionen an der Peripherie erfolgreich war. Doch Erfolge feiert er damit "nur“ bei einem eindeutig definierten Elektorat (wie die Wahlen von 2017 zeigen). Unsere Ergebnisse bestätigen, dass der politische Wandel in der Tschechischen Republik nach wie vor geographisch bedingt ist.

Man könnte geneigt sein, die Existenz einiger soziogeographischer Prozesse zu betrachten, die dem Parteienwettbewerb zu Grunde liegen (konkret [oder beispielsweise] die hohe Permanenz soziohistorischer Muster des Wählerverhaltens). Diese können nicht mit einem zentrierten und technokratischen populistischen 
Appell überwunden werden. Und auch die geographische Konzentration der Partisanenwahlen in Tschechien bestätigte den allgemeinen Trend einer zunehmenden Polarisierung der Wählerschaft (Jennings and Stoker 2016; Johnston/Manley/Jones 2016), die eine eigene räumliche Dimension hat. Insgesamt können wir auch in der Tschechischen Republik von einer Spaltung zwischen Wählerpräferenzen in den entwickelten Gebieten und den strukturell benachteiligten Regionen sprechen, ${ }^{5}$ aber es stellt sich die Frage, ob es der ANO-Bewegung nochmals gelingen kann, ihre heutige Wählerschaft bei Bedarf fast komplett auszutauschen oder ob dieser Wandel, wie wir ihn bei den Wahlen von 2013 und 2017 darstellen konnten, zukünftig nicht mehr möglich ist.

\section{Literatur}

Arditi, Benjamin (2004), Populism as a spectre of democracy: a response to Canovan, in: Political Studies, Vol. 52(I), I35-I43.

Arditi, Benjamin (2010), Populism is hegemony is politics? On Ernesto Laclau's On Populist Reason, in: Constellations, Vol. I7(3), 488-497.

Anselin, Luc (1995), Local Indicators of Spatial Association-LISA, in: Geographical Analysis, Vol. 27(2), 93-II5.

Balík, Stanislav/Vít Hloušek (2016), The development and transformation of the Czech party system after I989, in: Acta Politologica, Vol. 8(2), IO3-II7.

Bickerton, Christopher (2017), Populism and technocracy: opposites or complements?, in: Critical Review of International and Political Philosophy, Vol. 20(2), 186-206.

Bickerton, Christopher/Carlo Invernizzi Acceti (2018), 'Techno-populism' as a new party family: the case of the Five Star Movement and Podemos, in: Contemporary Italian Politics, Vol. IO(2), I32-I5O.

Bernard, Josef/Martin Šimon (20I4), Prostorové vzorce volebního chování v letech 1992-2006: popis, proměny a možné prričiny, in: Kostelecký, Tomáš (Hrsg.), Koho volí Vaši sousedé? Prostorové vzorce volebního chování na území Česka od roku 1920 do roku 2006, jejich změny a možné přičiny, Praha: SLON, IO9-I32.

Bernard, Josef/Tomáš Kostelecký/Martin Šimon (20I4), Existují prostorové kontextové vlivy na volební chování iv relativně nacionalizovaném stranickém systému? Př́iklad Česka, in: Geografie, Vol. II9(3), 240-258.

Bernard, Josef/Martin Šimon (20I7), Vnitřní periferie v Česku: Multidimenzionalita sociálního vyloučení ve venkovských oblastech, in: Sociologický časopis, Vol. 53(I), 3-28.

5 Im Übrigen fand auch der Erfolg der Tschechischen Piratenpartei von 2017 vor allem in den traditionellen liberal-konservativen Regionen statt, s. Maškarinec (2020).
De Blasio, Emiliana/Michele Sorice (2018), Populism between direct democracy and the technological myth, in: Palgrave Communications, Vol. 4(I), I-II.

Brunnerová, Olga (2019), Not all political entrepreneurs are created equal: The institutionalisation of entrepreneurial parties in Central Europe, in: Central European Journal of Politics, Vol. 5(I), IOO-I24.

Buštíková, Lenka/Petra Guasti (2018), The State as a Firm: Understanding the Autocratic Roots of Technocratic Populism, in: East European Politics and Societies, Vol. 33(2), 302-330.

Canovan, Margaret (1999), Trust the people! Populism and the two faces of democracy, in: Political Studies, Vol. 47(I), 2-I6.

Canovan, Margaret (2004), Populism for Political Theorists?, in: Journal of Political Ideologies, Vol. 9(3), 24I252.

CVVM (2019), Důvěra $\mathrm{k}$ vybraným institucím veřejného života - září 20I9, Internet: https://cvvm.soc.cas.cz/ media/com_form2content/documents/c2/a5oli/f9/ poigloo8.pdf (Zugriff: 20.03. 2020).

Fischer, Frank (1990), Technocracy and the Politics of Expertise, Newbury Park, CA: Sage.

Fotheringham, A. Stewart/Chris Brunsdon/Martin Charlton (2002), Geographically Weighted Regression: the analysis of spatially varying relationships, Chichester: John Wiley \& Sons.

Hanley, Sean/Milada Anna Vachudová (2018), Understanding the illiberal turn: democratic backsliding in the Czech Republic, in: East European Politics, Vol. 34(3), 276-296.

Havlik, Vlastimil/Petr Voda (2016), The Rise of New Political Parties and Re-Alignment of Party Politics in the Czech Republic, in: Acta Politologica, Vol. 8(2), II9-I44.

Havlik, Vlastimil/Petr Voda (2018), Cleavages, Protest or Voting for Hope? The Rise of Centrist Populist Parties in the Czech Republic, in: Swiss Political Science Review, Vol. 24(2), I6I-I86.

Hloušek, Vít/Lubomír Kopeček (2008), Cleavages in the Contemporary Czech and Slovak Politics Between Persistence and Change, in: East European Politics and Societies: and Cultures, Vol. 22(3), 518-552.

Kenneally, Ivan (2009), Technocracy and Populism, in: The New Atlantis, Vol. 24, 46-60.

Kaltwasser, Cristóbal Rovira (2012), The ambivalence of Populism: Threat and Corrective for Democracy, in: Democratization, Vol. 19(2), I84-208.

Kopeček, Lubomír (2016), I'm Paying, So I Decide": Czech ANO as an Extreme Form of a Business-Firm Party, in: East European Politics and Societies, Vol. 30(4), 725749.

Kostelecký, Tomáš/Josef Bernard (2OI4), Závěr, in: Kostelecký, Tomáš (Hrsg.), Koho volí Vaši sousedé? Prostorové vzorce volebního chování na území Česka od roku 
I920 do roku 2006, jejich změny a možné př́činy, Praha: SLON, I39-I43.

Linek, Lukáš/Pat Lyons (2013), Dočasná stabilita? Volební podpora politických stran v České republice v letech I990-20IO, Praha: Sociologické nakladatelství.

Maškarinec, Pavel (20I3), Prostorová analýza prezidentských voleb v České republice v roce 2013, in: Sociológia, Vol. 45(5), 435-469.

Maškarinec, Pavel (2017), A spatial analysis of Czech parliamentary elections, 2006-2013, in: Europe-Asia Studies, Vol. 69(3), 426-457.

Maškarinec, Pavel (2020), Crossing the left-right party divide? Understanding the electoral success of the Czech Pirate Party in the 2017 parliamentary elections, in: Politics $4 \mathrm{O}(4), 510-526$.

Mouffe, Chantal (2005), On the political, Abingdon: Routledge.

Mouffe, Chantal (2016), The Populist Moment, Internet: https://www.opendemocracy.net/democraciaabierta/chantal-mouffe/populist-moment (Zugriff: 15.06. 2020).

Mudde, Cas (2004), The Populist Zeitgeist, Government and Opposition, Vol. 39(4), 54I-563.

Müller, Jan-Werner (2016), Was ist Populismus? - Ein Essay, Frankfurt: suhrkamp.

Novotný, Lukáš (2015), Die deutsch-tschechischen Beziehungen und die Präsidentschaftswahl 2013, in: Österreichische Zeitschrift für Politikwissenschaft, Vol. $44(4), 12-24$.

Novotný, Lukáš (2017), Der allgegenwärtige Schatten des Kommunismus im tschechischen Parteiensystem, in: Österreichische Zeitschrift für Politikwissenschaft, Vol. 46(2), I7-26.

Novotný, Lukáś/Daniel Šárovec (2020), Ich will Tschechien wie ein Unternehmen führen, in: Zeitschrift für Politik, Vol. 67(3), 314-334.

Pink, Michal/Otto Eibl (2018), The 2016 Czech regional elections: Without real regional parties or voters' interest, in: Regional and Federal Studies, Vol. 28(3), 353365 .

Rooduijn, Matthijs (20I4), Vox Populismus: A Populist Radical Right Attitude among the Public?, in: Nations and Nationalism, Vol. 2O(I), 80-92.

Sax, Ondřej (20I8), Volební geografie, in: Šedo, Jakub (Hrsg.), České prezidentské volby v roce 20I3. Jiný souboj, stejný vítěz, Brno: CDK, 175-206.

Shin, Michael E./John Agnew (2007), The geographical dynamics of Italian electoral change, 1987-200I, in: Electoral Studies, Vol. 26(2), 287-302.

Stanley, Ben (2008), The Thin Ideology of Populism, in: Journal of Political Ideologies, Vol. I3(I), 95-IIO.

Śárovec, Daniel (2017), Krajské volby 2016 a nové politické strany v České republice, in: Central European Journal of Politics, Vol. 3(2), I-25.
Tobler, W. R. (1970), A Computer Movie Simulating Urban Growth in the Detroit Region, in: Economic Geography, Vol. 46(2), 234-240.

Unwin, A./D. Unwin (1998), Exploratory Spatial Data Analysis with Local Statistics, in: The Statistician, Vol. 47(3), 415-42I.

de Vreese, Claes/Frank Esser/Toril Aalberg (2018), Populism as an Expression of Political Communication Content and Style: A New Perspective, in: The International Journal of Press/Politic, Vol. 23(4), 423-438.

Welt.de (2017), Wie ein Anti-Europäer Brüssel abkassiert, Internet: https://www.welt.de/wirtschaft/articleI69929309/Wie-ein-Anti-Europaeer-Bruesselabkassiert.html (Zugriff: 20.03. 2020).

\section{Autoren}

Pavel Maškarinec ist Dozent am Institut für Politikwissenschaft der Philosophischen Fakultät der JanEvangelista-Purkyně Universität in Ústí nad Labem $(\mathrm{CZ})$. Seine Arbeits- und Forschungsschwerpunkte umfassen Raumanalysen von Wahlen, quantitative Erforschung des WählerInnenverhaltens sowie der Wahlsysteme.

Lukáš Novotný ist Dozent am Institut für Politikwissenschaft der Philosophischen Fakultät der JanEvangelista-Purkyně Universität in Ústí nad Labem (CZ). Er publiziert und forscht zu Themen der Parteienforschung sowie der öffentlichen Politiken und der Regionalentwicklung. 
